\title{
Acesso aberto a artigos, dados e materiais de pesquisa na RBMFC
}

\section{Open access to articles, data and research materials on RBMFC}

\section{Acceso abierto a artículos, datos y materiales de investigación en RBMFC}

\author{
Leonardo Ferreira Fontenelle ${ }^{1}{ }^{\circledR}$,Thiago Dias Sarti ${ }^{1}$ \\ ${ }^{1}$ Revista Brasileira de Medicina de Família e Comunidade (RBMFC) - Rio de Janeiro, RJ, Brasil.
}

\section{Resumo}

Acesso aberto aos artigos, dados e materiais de pesquisa são alguns elementos-chave da ciência aberta, um movimento plural que visa a transformar a criação e a comunicação do conhecimento científico. Graças à publicação de artigos em acesso aberto e à adoção de boas práticas editoriais, a RBMFC tem seus artigos indexados na LILACS e, mais recentemente, no DOAJ. A RBMFC também adota as diretrizes Transparency and Openness Promotion do Center for Open Science, e disponibiliza suas referências na CrossRef em domínio público, graças ao que a revista foi incluída no índice de citações OpenCitations. Com isso, a RBMFC espera tornar mais acessível, democrática e eficiente a pesquisa em medicina de família e comunidade e atenção primária à saúde.

Palavras-chave: Comunicação Acadêmica; Políticas Editoriais; Publicação de Acesso Aberto; Metadados; Revisão da Pesquisa por Pares

\begin{abstract}
Open access to research articles, data and materials are some key elements of open science, a plural movement that aims to transform the creation and communication of scientific knowledge. Thanks to the publication of articles in open access and the adoption of good editorial practices, RBMFC has its articles indexed in LILACS and, more recently, in DOAJ. RBMFC also adopts the Transparency and Openness Promotion guidelines from the Center for Open Science, and makes its references in CrossRef available in the public domain, thanks to which the journal was included in the OpenCitations citation index. With this, RBMFC hopes to make research in family and community medicine and primary health care more accessible, democratic and efficient
\end{abstract}

Keywords: Scholarly Communication; Editorial Policies; Open Access Publishing; Metadata; Peer Review, Research

Como citar: Fontenelle LF, Sarti TD. Acesso aberto a artigos, dados e materiais de pesquisa na RBMFC. Rev Bras Med Fam Comunidade. 2020;15(42):2671. https://doi.org/10.5712/rbmfc15(42)2671

\author{
Autor correspondente: \\ Leonardo Ferreira Fontenelle. \\ E-mail: leonardof@leonardof.med.br \\ Fonte de financiamento: \\ declaram não haver. \\ Parecer CEP: \\ não se aplica. \\ Procedência: \\ encomendado. \\ Avaliação por pares: \\ interna. \\ Recebido em: 11/08/2020. \\ Aprovado em: 11/08/2020.
}




\section{Resumen}

El acceso abierto a artículos, datos y materiales de investigación son algunos de los elementos clave de la ciencia abierta, un movimiento plural que tiene como objetivo transformar la creación y comunicación del conocimiento científico. Gracias a la publicación de artículos en acceso abierto y la adopción de buenas prácticas editoriales, RBMFC tiene sus artículos indexados en LILACS y, más recientemente, en DOAJ. RBMFC también adopta las directrices Transparency and Openness Promotion del Center for Open Science, y hace que sus referencias en CrossRef estén disponibles en el dominio público, gracias a lo cual la revista fue incluida en el índice de citas de OpenCitations. Con esto, RBMFC espera que la investigación en medicina familiar y comunitaria y atención primaria de salud sea más accesible, democrática y eficiente.

Palabras clave: Comunicación Académica; Políticas Editoriales; Publicación de Acceso Abierto; Metadatos; Revisión de la Investigación por Pares

A produção e a disseminação de conhecimento andam de mãos dadas. A criação das primeiras revistas científicas, por exemplo, esteve associada à revolução científica do século XVII, quando a produção de conhecimento científico foi profissionalizada e institucionalizada. Com o advento da Internet, as revistas científicas e índices bibliográficos passaram a ser acessadas através da Web, mas as mudanças não pararam aí. Graças à Web 2.0, a produção do conhecimento científico está cada vez mais aberta, tanto entre os pesquisadores quanto para o público geral. ${ }^{1}$

Talvez um dos aspectos mais importantes da ciência aberta seja o acesso aberto aos produtos de pesquisa, como os artigos científicos, dados de pesquisa, materiais de pesquisa e código das análises estatísticas. A lógica do acesso aberto é que não faz sentido utilizar recursos públicos para adquirir acesso aos produtos de pesquisa, uma vez que essas pesquisas foram financiadas com recursos públicos. A abertura do acesso inclui não apenas o direito irrevocável a consultar esses produtos de pesquisa, mas também a redistribuí-los e de reutilizá-los de outras formas. ${ }^{2}$

A maioria dos artigos científicos já são publicados em acesso aberto. ${ }^{3}$ Trata-se de um fenômeno mundial, com maior destaque para países de baixa renda e para a África Subsaariana. ${ }^{4}$ Nos Estados Unidos e Europa, o avanço do acesso aberto se deve muito às agências de fomento à pesquisa, como o National Institutes of Health dos Estados Unidos e as organizações que hoje constituem o cOALition S. ${ }^{5} \mathrm{Na}$ América Latina, a escassez de recursos para assinar revistas científicas levou a um modelo de comunicação científica liderado por pesquisadores (em vez de editoras comerciais), no qual as revistas científicas são publicadas sem fins comerciais e o acesso aberto foi adotado precocemente, como forma de aumentar o alcance da produção científica local. ${ }^{6}$

O acesso aberto na Revista Brasileira de Medicina de Família e Comunidade (RBMFC) ecoa em muito seu contexto latino-americano. Quando a revista era impressa, sua distribuição era um benefício da associação à Sociedade Brasileira de Medicina de Família e Comunidade (SBMFC), ${ }^{7}$ mas a assinatura custava apenas $R \$ 48,00$ para os não associados, e os autores não precisavam abrir mão do copyright para publicar seus artigos na revista. Assim que a RBMFC migrou para uma plataforma online, a revista adotou explicitamente uma licença da Creative Commons para seus artigos. ${ }^{8}$

Foi graças à sua publicação em acesso aberto, e à sua adoção de boas práticas editoriais, que a RBMFC foi indexada no Directory of Open Access Journals (DOAJ) ${ }^{8}$ e, mais tarde, na LILACS (Literatura Latino-Americana e do Caribe em Ciências da Saúde), que desde agosto de 2008 exige acesso aberto para a inclusão de periódicos. A RBMFC chegou a perder o prazo de recadastramento no DOAJ devido a uma transição de gestão, mas neste ano a revista solicitou novamente a inclusão e foi atendida. 
Essa nova inclusão da RBMFC no DOAJ é ainda mais significativa do que a primeira, por uma série de motivos. Em primeiro lugar, essa nova inclusão sinaliza que a revista atende aos Principles of Transparency and Best Practice in Scholarly Publishing, ${ }^{9}$ desenvolvidos pelo DOAJ com o COPE (Committee on Publication Ethics), OASPA (Open Access Scholarly Publishers Association) e WAME (World Association of Medical Editors). Em segundo lugar, desta vez a RBMFC não está sendo meramente listada junto a outros periódicos, mas sim indexada em nível de artigo (assim como acontece na LILACS). Por fim, desta vez a RBMFC foi certificada com o DOAJ Seal of Approval for Open Access Journals, uma "marca de certificação concedida pelo DOAJ a periódicos que atingem alto nível de abertura, adesão a boas práticas e altos padrões de publicação." (https://doaj.org/faq\#seal).

Mas o acesso aberto aos artigos científicos é apenas uma das facetas da ciência aberta na RBMFC. Seguindo as diretrizes do Transparency and Openness Promotion do Center for Open Science,${ }^{10}$ a revista estimula seus autores a compartilhar dados anonimizados e materiais de pesquisa (tais como questionários e código de análises estatísticas), ${ }^{11}$ e a citá-los em seus artigos. ${ }^{12}$ A revista também aceita submissões que já estejam disponíveis em servidores de preprint, efetivamente estimulando seus autores a acelerar a comunicação científica.

A própria RBMFC disponibiliza abertamente seus metadados, ou seja, os dados sobre seus artigos. Qualquer revista compartilha o título dos artigos, autoria e outros metadados com índices bibliográficos, mas a RBMFC disponibiliza a qualquer um, seguindo o protocolo OAI-PMH (Open Archives Initiative Protocol for Metadata Harvesting). Além disso, neste ano a RBMFC passou a incluir a lista de referências dos artigos ao depositar os metadados junto à CrossRef para obter o DOI (identificador de objetos digitais) dos artigos. Em acesso aberto, naturalmente.

Depositar as referências em acesso aberto é essencial para que as métricas de produção acadêmica sejam baseadas em dados abertos, não proprietários. ${ }^{13-15}$ Com a notável exceção da Elsevier (e American Chemical Society, IEEE, Wolters Kluwer Health), as grandes editoras acadêmicas já depositam metadados de suas publicações na CrossRef em acesso aberto, aderindo à Initiative for Open Citations (I4OC). ${ }^{14,16}$ Enquanto a CAPES (Coordenação de Aperfeiçoamento de Pessoal de Nível Superior) não começa a aproveitar esses dados em suas avaliações, as referências de artigos da RBMFC já podem ser consultadas através das plataformas OpenCitations e Dimensions, ou através do aplicativo de bibliometria VOSViewer.

Em acesso aberto ou não, depositar as referências junto à CrossRef também habilita a RBMFC a utilizar seu serviço "Cited-by". ${ }^{17}$ Assim que nossa plataforma eletrônica (OJS) permitir, a revista deverá utilizar esse serviço para listar, na página dos artigos, uma lista dos objetos digitais (artigos, livros, etc.) que os citam.

Em resumo, a RBMFC publica seus artigos em acesso aberto e disponibiliza em acesso aberto os metadados sobre esses mesmos artigos; também promove o depósito de dados e materiais de pesquisa (preferencialmente em acesso aberto) em repositórios de dados como Open Science Framework (OSF) e Zenodo, bem como a citação desses dados e materiais em seus artigos. Isso resulta em maior visibilidade da pesquisa publicada na RBMFC e no aprimoramento do processo científico como um todo.

Este editorial focou no acesso aberto, que é uma parte importante, mas não é toda a ciência aberta. Estamos sempre estudando novas formas de abrir a produção e disseminação de conhecimento científico, por exemplo através da revisão por pares aberta. Estamos analisando os dados de uma enquete realizada 
junto às partes interessadas (leitores, autores e revisores, bem como sócios da SBMFC). ${ }^{18}$ Em breve os dados de pesquisa, questionário e código de análise estatística estarão disponíveis em https://osf.io/u9p4n, e o manuscrito estará disponível em um repositório de preprints.

\section{Conflitos de interesses}

Os autores são editores-chefe da Revista Brasileira de Medicina de Família e Comunidade (RBMFC)

\section{REFERÊNCIAS}

1. Bartling S, Friesike S. Towards another scientific revolution. In: Bartling S, Friesike S, eds. Opening science. Cham: Springer International Publishing; 2014. p. 3-15. DOI: https://doi.org/10.1007/978-3-319-00026-8_1

2. Sitek D, Bertelmann R. Open access: a state of the art. In: Bartling S, Friesike S, eds. Opening science. Cham: Springer International Publishing; 2014. p. 139-53. DOI: https://doi.org/10.1007/978-3-319-00026-8_9

3. Hook D, Hahnel M, Calvert I.The ascent of open access. Digital Science. 2019 Jan. DOI: https://doi.org/10.6084/m9.figshare.7618751.v2

4. Iyandemye J, Thomas MP. Low income countries have the highest percentages of open access publication: a systematic computational analysis of the biomedical literature. PLoS ONE. 2019 Jul;14(7):e0220229. DOI: https://doi.org/10.1371/journal.pone.0220229

5. Wise A, Estelle L. How society publishers can accelerate their transition to open access and align with Plan S. Learned Publishing. 2020 Jan;33(1):14-27. DOI: https://doi.org/10.1002/leap.1272

6. Alperin JP. The public impact of Latin America's approach to open access [thesis]. Stanford, California: Stanford University; 2015.

7. Anderson MIP, Falk JW. Editorial. Rev Bras Med Fam Comunidade. 2004 Nov; 1(1):1. DOI: https://doi.org/10.5712/rbmfc1(1)1

8. Demarzo MMP, Milhomens DM. RBMFC em 2011: evolução e desafios. Rev Bras Med Fam Comunidade. 2011;6(21):227-9. DOI: https:// doi.org/10.5712/rbmfc6(21)499

9. Committee on Publication Ethics (COPE). Principles of transparency and best practice in scholarly publishing. Hampshire, UK: COPE; 2018. DOI: https://doi.org/10.24318/cope.2019.1.12

10. Nosek BA, Alter G, Banks GC, Borsboom D, Bowman SD, Breckler SJ, et al. Promoting an open research culture. Science. 2015 Jun;348(6242):1422-5. DOI: https://doi.org/10.1126/science.aab2374

11. Fontenelle LF, Sarti TD. Pesquisar para quê?. Rev Bras Med Fam Comunidade. 2020 Jan;15(42):2319. DOI: https://doi.org/10.5712/ rbmfc15(42)2369

12. Fontenelle LF, Sarti TD. Renovação das práticas editoriais e instruções aos autores da RBMFC a partir de 2019. Rev Bras Med Fam Comunidade. 2019 Jun;14(41):1963. DOI: https://doi.org/10.5712/rbmfc14(41)1963

13. Shotton D. Publishing: open citations. Nature. 2013 Out;502(7471):295-7. DOI: https://doi.org/10.1038/502295a

14. Shotton D. Funders should mandate open citations. Nature. 2018 Jan;553:129. DOI: https://doi.org/10.1038/d41586-018-00104-7

15. Chawla DS. Now free: citation data from 14 million papers, and more might come. Science. 2017 Abr. DOI: http://dx.doi.org/10.1126/ science.aal1012

16. Heibi I, Peroni S, Shotton D. Software review: COCI, the OpenCitations Index of Crossref open DOI-to-DOI citations. Scientometrics. 2019 Set;121(2):1213-28. DOI: https://doi.org/10.1007/s11192-019-03217-6

17. Lammey R. CrossRef developments and initiatives: an update on services for the scholarly publishing community from CrossRef. Sci Ed. 2014;1(1):13-8. DOI: https://doi.org/10.6087/kcse.2014.1.13

18. Fontenelle LF, Sarti TD. Attitudes to open peer review among stakeholders of RBMFC. OSF. 2020. DOI: https://doi.org/10.17605/OSF.IO/ MVC98 